\title{
An almost linear time algorithm for finding Hamilton cycles in sparse random graphs with minimum degree at least three.
}

\author{
Alan Frieze* and Simi Haber \\ Department of Mathematical Sciences, \\ Carnegie Mellon University, \\ Pittsburgh PA15217.
}

October 12, 2018

\begin{abstract}
We describe an algorithm for finding Hamilton cycles in random graphs. Our model is the random graph $G=G_{n, m}^{\delta \geq 3}$. In this model $G$ is drawn uniformly from graphs with vertex set $[n], m$ edges and minimum degree at least three. We focus on the case where $m=c n$ for constant $c$. If $c$ is sufficiently large then our algorithm runs in $O\left(n^{1+o(1)}\right)$ time and succeeds w.h.p.
\end{abstract}

\section{Introduction}

The threshold for the existence of Hamilton cycles in random graphs has been known very precisely for some time, Komlós and Szemerédi [15, Bollobás [4], Ajtai, Komlós and Szemerédi [1. Computationally, the Hamilton cycle problem is one of the original NP-complete problems described in the paper of Karp [13. On the other hand Angluin and Valiant 2] were the first to show that the Hamilton cycle problem could be solved efficiently on random graphs. The algorithm in [2] is randomised and very fast, $O\left(n \log ^{2} n\right)$ time, but requires $K n \log n$ random edges for sufficiently large $K>0$. Bollobás, Fenner and Frieze [7] gave a deterministic polynomial time algorithm that works w.h.p. at the exact threshold for Hamiltonicity, it is shown to run in $O\left(n^{3+o(1)}\right)$ time.

The challenge therefore is to find efficent algorithms for graphs with a linear number of edges. Here we have to make some extra assumptions because a random graph with $c n$ edges is very unlikely to be Hamiltonian. It will have isolated vertices. It is natural therefore to consider models of random graphs with a linear number of edges and minimum degree $\delta$ at least two. In fact minimum degree three is required to avoid the event of having three vertices of degree two having a common neighbor. For example, in the case of random $r$-regular graphs, $r=O(1) \geq 3$, Robinoson and Wormald [17, [18] settled the existence question and Frieze, Jerrum, Molloy, Robinson and Wormald [1] gave a polynomial time algorithm for finding a Hamilton cycle. The running time of this algorithm was not given explicitly, but it is certainly $\Omega\left(n^{3}\right)$.

We will work on a model where the assumption is that $\delta \geq 3$ as opposed to all vertices having degree exactly three. It is tempting to think that existence results for the regualr case $r=3$ will help. Unfortunately, this is not true. The random graph $G_{n, m}^{\delta \geq 3}$ is uniformly sampled from the set $\mathcal{G}_{n, m}^{\delta \geq 3}$ of graphs with vertex set $[n]$, $m$ edges and minimum degree at least three.

Frieze, 9] gave an $O\left(n^{3+o(1)}\right)$ time algorithm for finding large cycles in sparse random graphs and this can be adapted to find Hamilton cycles in $G_{n, c n}^{\delta \geq 3}$ in this time for sufficiently large $c$. The paper [10] gives an

\footnotetext{
*Research supported in part by NSF Grant CCF2013110
} 
algorithm that reduces this to $n^{1.5+o(1)}$ for sufficiently large $c$. The main aim of this paper is to construct an almost linear time algorithm for this model.

Theorem 1.1. If $c$ is sufficiently large then our algorithm finds a Hamilton cycle in $G_{n, m}^{\delta \geq 3}, m=c n$, and runs in $O\left(n^{1+o(1)}\right)$ time and succeeds w.h.p.

Remark 1.1. The $n^{o(1)}$ term here is $(\log n)^{O(\log \log n)}$ which is tantalisingly close to best possible(?) $\log { }^{O(1)} n$.

\section{Outline of the paper}

The paper [8] gave an efficient algorithm for finding the maximum matching in a sparse random graph. Its approach was to (i) use the simple greedy algorithm of Karp and Sipser 14] and then (ii) augment it to a maximum matching using alternating paths. In this paper we replace the Karp-Sipser algorithm with the algorithm 2GREEDY that w.h.p. finds a 2-matching in $G=G_{n, m}^{\delta \geq 3}$ with $O(\log n)$ components and we replace alternating paths with extensions and rotations. (A 2-matching is a spanning subgraph of maximum degree at most two).

In Section 3 we will describe our algorithm. We will describe it in two subsections. We will describe 2GREEDY for finding a good 2-matching $M$ in detail in Section 3.1. In section 3.2 we will describe an algorithm ExTENDROTATE that uses extensions and rotations to convert $M$ into a Hamilton cycle. In Section 4 we discuss some "residual randomness" left over by 2 GREEDY. In Section 5 we prove some structural properties of $G_{n, m}^{\delta \geq 3}$. In Section 6 we prove some properties relating the output of 2GREEDY to the execution of EXTEND-ROTATE. In Section 7 we do a final calculation to finsih the proof. In Section 8 we point to our difiiculties in proving $n \log ^{O(1)} n$ and in Section 9 we make some final remarks.

\section{Algorithm}

As already stated, there are two phases to the algorithm. First we find a good 2-matching $M$ and then we convert it to a Hamilton cycle. We look first at how we find $M$.

\subsection{Algorithm 2GREEDY}

We greedily and randomly choose edges to add to $M$. Edges of $M$ are deleted from the graph. We let $b(v) \in\{0,1,2\}$ denote the degree of $v$ in $M$. Once $b(v)=2$ its incident edges are no longer considered for selection. The vertex itself is deleted from the graph. Thus the graph from which we select edges will shrink as the algorithm progresses. We will use $\Gamma$ to denote the current subgraph from which edges are to be selected. When there are vertices $v$ of degree $2-b(v)$ (or less) in $\Gamma$, we take care to choose an edge incident with such a vertex. Our observation being that there is a maximum cardinality 2-matching of $\Gamma$ that contains such an edge.

If every vertex $v$ of $\Gamma$ had degree at least $3-b(v)$ then we choose an edge randomly from edges that are incident with vertices $v$ that have $b(v)=0$. In this way, we quickly arrive at a stage where every vertex of $\Gamma$ has $b(v)=1$. At this point we use the algorithm of $\left[8\right.$ to find a (near) perfect matching $M^{*}$, which we add to $M$ as our final 2-matching.

We describe 2GREEDY in enough detail to make some of its claimed properties meaningful.

We let

- $\mu$ be the number of edges in $\Gamma$,

- $Y_{k}=\left\{v \in[n]: d_{\Gamma}(v)=k\right.$ and $\left.b(v)=0\right\}, k=0,1,2$, 
- $Z_{k}=\left\{v \in[n]: d_{\Gamma}(v)=k\right.$ and $\left.b(v)=1\right\}, k=0,1$,

- $Y=\left\{v \in[n]: d_{\Gamma}(v) \geq 3\right.$ and $\left.b(v)=0\right\}$,

- $Z=\left\{v \in[n]: d_{\Gamma}(v) \geq 2\right.$ and $\left.b(v)=1\right\}$,

- $M$ is the set of edges in the current 2-matching.

Note that $V(\Gamma)=[n] \backslash\left(Y_{0} \cup Z_{0}\right)$ and that $b(v) \in\{0,1\}$ for $v \in V(\Gamma)$.

We will assume that the input to our algorithm is an ordered sequence $\sigma_{m}=\left(e_{1}, e_{2}, \ldots, e_{m}\right)$ where $m=c n$. Here $E_{m}=\left\{e_{1}, e_{2}, \ldots, e_{m}\right\}$ are the edges of $G_{n, m}^{\delta \geq 3}$ and $\sigma_{m}$ is a random ordering of $E_{m}$. Once these orderings are given, the vertices and edges are processed in a deterministic fashion. Thus for example, if 2GREEDY requires a random edge with some property, then it is required to take the first available edge in the given ordering.

We now give details of the steps of

\section{Algorithm 2GREEDY:}

Step $1\left(\right.$ a) $Y_{1} \neq \emptyset$

Choose $v \in Y_{1}$. We choose $v$ by finding the first edge in the ordering $\sigma$ that contains a member of $Y_{1}$. Suppose that its neighbour in $\Gamma$ is $w$. We delete the edge $(v, w)$ from $\Gamma$ add $(v, w)$ to $M$ and move $v$ to $Z_{0}$.

(i) If $w$ is currently in $Y$ then move it to $Z$. If it is currently in $Y_{1}$ then move it to $Z_{0}$. If it is currently in $Y_{2}$ then move it to $Z_{1}$. Call this re-assigning $w$.

(ii) If $b(w)=1$ then we move $w$ to $Z_{0}$ and make the requisite changes due to the loss of other edges incident with $w$. Call this tidying up.

Step 1(b): $Y_{1}=\emptyset$ and $Y_{2} \neq \emptyset$

Choose $v \in Y_{2}$. We choose $v$ by finding the first edge in the ordering $\sigma$ that contains a member of $Y_{2}$. Suppose that its neighbours in $\Gamma$ are $w_{1}, w_{2}$.

We choose one of the neighbors at random, say $w_{1}$. We move $v$ to $Z_{1}$. We delete the edge $\left(v, w_{1}\right)$ from $\Gamma$ and place it into $M$. In addition,

(i) If $b\left(w_{1}\right)=0$ then put $b\left(w_{1}\right)=1$ and add the edge $\left(v, w_{1}\right)$ to $M$. Re-assign $w_{1}$.

(ii) If $b\left(w_{1}\right)=1$ then we delete $w_{1}$ from $\Gamma$. Tidy up.

Step 1(c): $Y_{2}=\emptyset$ and $Z_{1} \neq \emptyset$

Choose $v \in Z_{1}$. We choose $v$ by finding the first edge in the ordering $\sigma$ that contains a member of $Z_{1}$. Let $u$ be the other endpoint of the path $P$ of $M$ that contains $v$. Let $w$ be the unique neighbour of $v$ in $\Gamma$. We delete $v$ from $\Gamma$ and add the edge $(v, w)$ to $M$. In addition there are two cases.

(1) If $b(w)=0$ then we re-assign $w$.

(2) If $b(w)=1$ then we delete vertex $w$ and tidy up.

Step 2: $Y_{1}=Y_{2}=Z_{1}=\emptyset$ and $Y \neq \emptyset$

Choose the first edge $(v, w) \in E(\Gamma)$ in the order $\sigma_{m}$ incident with a vertex $v \in Y$. We delete the edge $(v, w)$ from $\Gamma$ and add it to $M$. We move $v$ from $Y$ to $Z$. There are two cases.

(i) If $b(w)=0$ then move $w$ from $Y$ to $Z$.

(ii) If $b(w)=1$ then we delete vertex $w$ and tidy up. 
Step 3: $Y_{1}=Y_{2}=Z_{1}=Y=\emptyset$

At this point $\Gamma$ will be distributed as $G_{\nu, \mu}^{\delta \geq 2}$ for some $\nu, \mu$ where $\mu=O(\nu)$. As such, it contains a (near) perfect matching $M^{*}[12$ and it can be found in $O(\nu)$ expected time [8]. This step comprises

Step 3a Apply the Karp-Sipser algorithm to $\Gamma$. W.h.p. this results in the construction of a matching $M_{1}^{*}$ that covers all but $\tilde{O}\left(\nu^{1 / 5}\right)$ vertices $U=\left\{u_{1}, u_{2}, \ldots, u_{\ell}\right\}$.

Step 3b Now find augmenting paths from $u_{2 i-1}$ to $u_{2 i}$ for $i \leq \ell / 2$. This produces the matching $M^{*}$.

The output of 2GREEDY is set of edges $M \leftarrow M \cup M^{*}$.

\subsection{Extension-Rotation Algorithm}

We now describe an algorithm, EXTEND-ROTATE that w.h.p. converts $M$ into a Hamilton cycle. The main idea is that of a rotation. Given a path $P=\left(u_{1}, u_{2}, \ldots, u_{k}\right)$ and an edge $e=\left(u_{k}, u_{i}\right)$ where $i \leq k-2$ we say that the path $P^{\prime}=\left(u_{1}, \ldots, u_{i}, u_{k}, u_{k-1}, \ldots, u_{i+1}\right)$ is obtained from $P$ by a rotation. $u_{1}$ is the fixed endpoint of this rotation. We say that $e$ is the inserted edge.

Given a path $P$ with endpoints $a, b$ we define a restricted rotation search $R R S(\nu)$ as follows: Suppose that we have a path $P$ with endpoints $a, b$. We start by doing a sequence of rotations with $a$ as the fixed endpoint. Furthermore

R1 We do these rotations in "breadth first manner", described in detail in Section 6.

R2 We stop this process when we have either (i) created $\nu$ endpoints or (ii) we have found a path $Q$ with an endpoint that has a neighbor $w$ outside of $Q$. The path $Q+w$ will be longer than $P$. We say that we have found an extension.

Let $\operatorname{END}(a)$ be the set of endpoints, other than $a$, produced by this procedure. Assuming that we did not find an extension and having constructed $\operatorname{END}(a)$, we take each $x \in E N D(a)$ in turn and starting with the path $P_{x}$ that we have found from $a$ to $x$, we carry out R1,R2 above with $x$ as the fixed endpoint and either find an extension or create a set of $\nu$ paths with $x$ as one endpoint and the other endpoints comprising a set $E N D(x)$ of size $\nu$.

Algorithm EXTEND-ROTATE

Step ER1 Let $K_{1}, K_{2}, \ldots, K_{r}$ be the components of $M$ where $\left|K_{1}\right|=\max \left\{\left|K_{j}\right|: j \in[r]\right\}$. If $K_{1}$ is a path then we let $P_{0}=K_{1}$, otherwise we let $P_{0}=K_{1} \backslash\{e\}$ where $e$ is any edge of $K_{1}$.

Step ER2 Let $P$ be the component of the current 2-matching $M$ that contains $P_{0}$. If $P$ is not a cycle, go directly to ER3. If $P$ is a Hamilton cycle we are done. Otherwise there is a vertex $u \in P$ and a vertex $v \notin P$ such that $f=(u, v)$ is an edge of $G$, assuming that $G$ is connected, see Lemma 6.3. Let $Q$ be the component containing $v$. By deleting an edge of $P$ incident to $u$ and (possibly) and edge of $Q$ incident with $v$ and adding $f$ we create a new path of length at least $|P|+1$ with vertex set equal to $V(P) \cup V(Q)$. Rename this path $P$.

Step ER3 Carry out $R S S(\nu)$ until either an extension is found or we have constructed $\nu$ endpoint sets.

Case a: We find an extension. Suppose that we construct a path $Q$ with endpoints $x, y$ such that $y$ has a neighbour $z \notin Q$.

(i) If $z$ lies in a cycle $C$ then let $R$ be a path obtained from $C$ by deleting one of the edges of $C$ incident with $z$. Let now $P=x, Q, y, z, R$ and go to Step ER2.

(ii) If $z=u_{j}$ lies on a path $R=\left(u_{1}, u_{2}, \ldots, u_{k}\right)$ where the numbering is chosen so that $j \geq k / 2$ then we let $P=x, Q, y, z, u_{j-1}, \ldots, u_{1}$ and go to Step ER2. 
Case b: If there is no extension then we search for an edge $f=(p, q)$ such that $p \in \operatorname{END}(a)$ and $q \in \operatorname{END}(p)$. If there is no such edge then the algorithm fails. If there is such an edge we let $Q$ be the corresponding path from $p$ to $q$. We replace $P$ in our 2-matching by the cycle $Q+f$ and go to ER2.

\subsection{Execution Time of the algorithm}

The expected running time of 2GREEDY is $O(n)$ and w.h.p. it completes in $O(n)$ time with a 2-matching $M$ with at most $K_{1} \log n$ components for some constant $K_{1}>0$. This follows from the results of [8] and [10].

To bound the execution time of EXTEND-ROTATE we first observe that it follows from [2] that $R S S(\nu)$ can be carried out in $O\left(\nu^{2} \log n\right)$ time. We will take

$$
\nu=n^{1 / 2+O(\varepsilon)}
$$

where

$$
\varepsilon=\frac{K(\log \log n)^{2}}{\log n}
$$

where $K$ is a sufficiently large positive constant and that $c$ is sufficiently large.

We now bound the number of executions of $R S S(\nu)$. Each time we execute Step ER3, we either reduce the number of components by one or we halve the size of one of the components not on the current path. So if the component sizes of $M_{0}$ are $n_{1}, n_{2}, \ldots, n_{\kappa}$ then the number of executions of Step ER3 can be bounded by

$$
\kappa+\sum_{i=1}^{\kappa} \log _{2} n_{i}=O\left(\log ^{2} n\right) .
$$

So the total execution time is w.h.p. of order

$$
n+\left(n^{1 / 2+O(\varepsilon)}\right)^{2} \log ^{2} n=O\left(n^{1+O(\varepsilon)}\right) .
$$

This clearly suffices for Theorem 1.1

We will now turn to discuss the probability that our algorithm succeeds after we have described 2GREEDY. We remind the reader that the analysis assumes that $c$ is sufficently large.

\section{Residual Randomness}

Let $G$ be a graph with an ordering of its edges and consider a run of 2 GREEDYon that graph. At every point of time each vertex is in one of the sets $Y_{0}, Z_{0}, Y_{1}, Y_{2}, Z_{1}, Y$ and $Z$ as defined above.

We let the set of vertices that were removed from the graph while in $Z$ be denoted by $R$. We call them "regular vertices". These vertices are removed from $\Gamma$ in the execution of a Step 1 or Step 2 of 2GREEDY and they are internal vertices of paths of $M$ at the start of Step 3.

For a vertex $v$ let $t_{v}$ be the time at which 2GREEDY deletes $v$ from $\Gamma$. Vertices $w$ that are not deleted before the start of Step 3 are given $t_{w}=\infty$. A vertex is early if $t_{v} \leq n^{1-\varepsilon}$ and late otherwise. An edge $e_{i}$ is punctual if $i \leq(1-\alpha) m$ and tardy otherwise, where $\alpha$ is a small positive constant.

When a vertex $v \in R$ gets matching degree two we take the incident non-matching edge $e$ with the lowest index in $\sigma$ to be its $Z$-witness. The fact that $v \in Z$ just before this happens implies that $e$ exists. We let $W$ denote the set of $Z$-witnesses. We next defne two sets $R_{0}$ and $\Lambda_{0}$ :

We let

$$
R_{0}=\{v \in R: v \text { is early and the } Z \text {-witness of } v \text { is punctual }\} .
$$


and

$$
\Lambda_{0}=\left\{v: v \text { has degree at least } 4 \text { in } \Gamma_{n^{1-\varepsilon}}\right\} .
$$

We may now state and prove the main lemma of this section.

Lemma 4.1. In what follows $R_{0}, \Lambda_{0}$ are defined with respect to $G$ and an ordreing of its edges. Let $e=\{x, y\}$ be a tardy edge of $G$ where $x \in R_{0}$ and $y \in \Lambda_{0}$. Let $G^{\prime}$ be the graph obtained from $G$ by deleting $e$. Assume that running 2GREEDY on $G$ up until Step 3 gives a 2-matching $M$ and a witness set $W$ and running 2GREEDY on $G^{\prime}$ up until Step 3 gives $M^{\prime}$ and $W^{\prime}$. Then $M=M^{\prime}$ and $W=W^{\prime}$.

Proof We claim that up to time $t_{x}$, 2GREEDY will delete the same vertices and edges from $\Gamma_{t}$ and $\Gamma_{t}^{\prime}$ and then delete $x$ from both. After this the two graphs will coincide and we are done. We do this by induction on $t$. This is clearly true for $t=0$ and assume that $\Gamma_{t}$ and $\Gamma_{t}^{\prime}$ differ only in $e$ and $t<t_{x}$. Note that the induction hypothesis implies that the sets $Y_{0}, Y_{1}, \ldots, Z$ are the same in $\Gamma_{t}, \Gamma_{t}^{\prime}$. This is because deleting edge $e$ does not affect $x$ 's status, because $e$ is after the $Z$-witness of $x$ in the order $\sigma$. It does not affect $y$ 's status, because the degree of $y$ will be at least 3 after the deletion.

Because the sets $Y_{0}, Y_{1}, \ldots, Z$ are unchanged, the choice of step is the same in $\Gamma_{t}$ and $\Gamma_{t}^{\prime}$. The difference between the two graphs only affects the degrees of $x$ and $y$ and by construction, this is never enough to change a choice of edge.

Remark 4.1. Suppose that $e_{i}=(v, w)$ and that (i) $v \in R_{0}$, (ii) $w \in \Lambda_{0}$ and (iii) $e_{i}$ is tardy. Then replacing $e_{i}$ by $\left(v^{\prime}, w^{\prime}\right)$ such that (i) $v^{\prime} \in R_{0}$ and (ii) $w^{\prime} \in \Lambda_{0}$ results in the the same output $M, W$.

The net effect of this is that if we condition on all edges except for the tardy edges between $R_{0}$ and $\Lambda_{0}$ then the unconditioned tardy $R_{0}: \Lambda_{0}$ edges are random. This is what we mean by there being residual randomness.

\section{$5 \quad$ Degree Sequence of $G_{n, m}^{\delta \geq 3}$}

The degrees of the vertices in $G$ are distributed as truncated Poisson random variables $\operatorname{Po}(\lambda ; \geq 3)$, see for example [3. More precisely we can generate the degree sequence by taking random variables $Z_{1}, Z_{2}, \ldots, Z_{n}$ where

$$
\mathbb{P}\left(Z_{i}=k\right)=\frac{\lambda^{k}}{k ! f_{3}(\lambda)} \quad \text { for } i=1,2, \ldots, n \text { and } k \geq 3,
$$

where $f_{j}(\lambda)=e^{\lambda}-\sum_{k=0}^{j-1} \frac{\lambda^{k}}{k !}$ for $j \geq 1$.

Then we condition on $Z_{1}+Z_{2}+\cdots Z_{n}=2 m$. The resulting $Z_{1}, Z_{2}, \ldots, Z_{n}$ can be taken to have the same distribution as the degrees of $G$. This follows from Lemma 4 of $[\underline{3}$. If we choose $\lambda$ so that

$$
\mathbb{E}(P o(\lambda ; \geq 3))=\frac{2 m}{n} \text { or } \frac{\lambda f_{2}(\lambda)}{f_{3}(\lambda)}=\frac{2 m}{n}
$$

then the conditional probability, $\mathbb{P}\left(Z_{1}+Z_{2}+\cdots Z_{\nu}=2 m\right)=\Omega(1 / \sqrt{n})$ and so we will have to pay a factor of $O(\sqrt{n})$ for removing the conditioning i.e. to use the simple inequality $\mathbb{P}(A \mid B) \leq \mathbb{P}(A) / \mathbb{P}(B)$. (This factor $O\left(n^{1 / 2}\right)$ can be removed but it will not be necessary to do this here).

The maximum degree $\Delta$ in $G$ is less than $\log n$ q.s 1 and equation (7) of [3] enables us to claim that that if $\nu_{k}, 2 \leq k \leq \log n$ is the number of vertices of degree $k$ then q.s.

$$
\left|\nu_{k}-\frac{n \lambda^{k} e^{-\lambda}}{k ! f_{3}(\lambda)}\right| \leq K_{1}\left(1+\sqrt{n \lambda^{k} e^{-\lambda} /\left(k ! f_{3}(\lambda)\right)}\right) \log n, 2 \leq k \leq \log n .
$$

for some constant $K_{1}>0$.

\footnotetext{
${ }^{1}$ A sequence of events, $\mathcal{E}_{n}$ occurs quite surely (q.s.) if $\mathbb{P}\left(\neg \mathcal{E}_{n}\right)=o\left(n^{-C}\right)$ for any $C>0$.
} 
In particular, this implies that if the degrees of the vertices in $G$ are $d_{1}, d_{2}, \ldots, d_{n}$ then q.s.

$$
\sum_{i=1}^{n} d_{i}\left(d_{i}-1\right)=O(n)
$$

Given the degree sequence we make our computations in the configuration model, see Bollobás [5]. Let $\mathbf{d}=\left(d_{1}, d_{2}, \ldots, d_{n}\right)$ be a sequence of non-negative integers with $2 m=c n$. Let $W=[2 \mathrm{cn}]$ be our set of points and let $W_{i}=\left[d_{1}+\cdots+d_{i-1}+1, d_{1}+\cdots+d_{i}\right], i \in[n]$, partition $W$. The function $\phi: W \rightarrow[n]$ is defined by $w \in W_{\phi(w)}$. Given a pairing $F$ (i.e. a partition of $W$ into $m=c n$ pairs) we obtain a (multi-)graph $G_{F}$ with vertex set $[n]$ and an edge $(\phi(u), \phi(v))$ for each $\{u, v\} \in F$. Choosing a pairing $F$ uniformly at random from among all possible pairings of the points of $W$ produces a random (multi-)graph $G_{F}$.

This model is valuable because of the following easily proven fact: Suppose $G \in \mathcal{G}_{n, \mathbf{d}}$, the set of (simple) graphs with vertex set $[n]$ and degree sequence $\mathbf{d}$. Then

$$
\mathbb{P}\left(G_{F}=G \mid G_{F} \text { is simple }\right)=\frac{1}{\left|\mathcal{G}_{n, \mathbf{d}}\right|} .
$$

It follows that if $G$ is chosen randomly from $\mathcal{G}_{n, \mathbf{d}}$, then for any graph property $\mathcal{P}$

$$
\mathbb{P}(G \in \mathcal{P}) \leq \frac{\mathbb{P}\left(G_{F} \in \mathcal{P}\right)}{\mathbb{P}\left(G_{F} \text { is simple }\right)} .
$$

Furthermore, applying Lemmas 4.4 and 4.5 of McKay [16] we see that if the degree sequence of $G$ satisfies (4) then $\mathbb{P}\left(G_{F}\right.$ is simple $)=\Omega(1)$. In which case the configuration model can substitute for $\mathcal{G}_{n, \mathbf{d}}$ (and hence $\left.G_{n, m}^{\delta \geq 3}\right)$ in dealing with events of probability $o\left(n^{-1 / 2}\right)$.

Lemma 5.1. W.h.p.

(a) $G_{n, m}^{\delta \geq 3}$ contains no set $S \subseteq[n], 3 \leq s=|S| \leq s_{0}=\frac{1}{5} \log _{c} n$ such that $S$ contains at least $s+1$ edges.

(b) Let $W_{1}$ denote the set of vertices $v$ that are within distance $\ell_{0}=2 \log \log n$ of a cycle of length at most $2 \ell_{0}$ in $G_{n, m}^{\delta \geq 3}$. Then w.h.p. $\left|W_{1}\right| \leq n^{1 / 2} \log ^{4 \ell_{0}} n$.

(c) W.h.p. there does not exist a connected subset of $K_{c} \log n \leq s \leq n^{3 / 5}$ vertices that contain s/10 vertices of degree at most 30 . Here $K_{c}$ is some sufficiently large constant.

Proof (a) The expected number of sets $S$ containing $|S|+1$ edges can be bounded by

$$
\begin{aligned}
& O\left(n^{1 / 2}\right) \sum_{s=3}^{s_{0}} \sum_{|S|=s} \sum_{D \geq 3 s} \sum_{\substack{d_{1}+\cdots+d_{s}=D \\
d_{1}, \ldots, d_{s} \geq 3}} \prod_{i=1}^{s} \frac{\lambda^{d_{i}}}{f_{3}(\lambda) d_{i} !}\left(\begin{array}{c}
D \\
s+1
\end{array}\right)\left(\frac{D}{c n-2 s}\right)^{s+1} \leq \\
& O\left(n^{1 / 2}\right) \sum_{s=3}^{s_{0}} \sum_{|S|=s} \sum_{D \geq 3 s}\left(\frac{D e}{s+1}\right)^{s+1}\left(\frac{D}{c n}\right)^{s+1} \frac{\lambda^{D} s^{D}}{D ! f_{3}(\lambda)^{s}} .
\end{aligned}
$$

Explanation: For (6) we choose a set of size $s$ with vertices of degree $d_{1}, d_{2}, \ldots, d_{s} \geq 3$ and $d_{1}+\cdots+d_{s}=D$. The term $\prod_{i=1}^{s} \frac{\lambda^{d_{i}}}{f_{3}(\lambda) d_{i} !}$ (modulo $O\left(n^{1 / 2}\right)$ ) accounts for the probability of these degrees. We then choose $s+1$ configuration points and approximate the probablity that they are all paired with other points associated with $s$ by $\left(\frac{D}{c n-2 s}\right)^{s+1}$. We use $\sum_{d_{1}+\cdots+d_{s}=D} \prod_{i=1}^{s} \frac{1}{d_{i} !}=\frac{s^{D}}{D !}$ to get (7).

Continuing we observe that $(D / c s)^{2 s+2} \leq\left(1+\frac{3}{c}\right)^{D}$ for $D \geq 3 s$. Thisis clearly true for $D \leq c s$ and follows by induction on $D \geq c s$. Therefore,

$$
\sum_{D \geq 3 s} \frac{D^{2 s+2} \lambda^{D} s^{D}}{D !} \leq(c s)^{2 s+2} e^{(\lambda+3) s} .
$$


Plugging this into (7) we get a bound of

$$
\begin{aligned}
& O\left(n^{1 / 2}\right) \sum_{s=3}^{s_{0}} \sum_{|S|=s} \frac{c e s^{2}}{n}\left(\frac{c e s^{2} e^{\lambda+3}}{n(s+1) f_{3}(\lambda)}\right)^{s} \\
\leq & O\left(\frac{c s^{2}}{n^{1 / 2}}\right) \sum_{s=3}^{s_{0}}\left(\frac{n e}{s}\right)^{s}\left(\frac{c e s^{2} e^{\lambda+3}}{n(s+1) f_{3}(\lambda)}\right)^{s} \\
\leq & O\left(\frac{c s^{2}}{n^{1 / 2}}\right) \sum_{s=3}^{s_{0}}\left(\frac{c e^{\lambda+3}}{f_{3}(\lambda)}\right)^{s} \\
\leq & O\left(\frac{c s^{2}}{n^{1 / 2}}\right) \sum_{s=3}^{s_{0}}\left(2 c e^{3}\right)^{s} \\
= & O(1) .
\end{aligned}
$$

(b)

$$
\mathbb{E}\left(\left|W_{1}\right|\right) \leq O\left(n^{1 / 2}\right) \sum_{k \leq \ell_{0}, \ell \leq 2 \ell_{0}}\left(\begin{array}{c}
n \\
k+\ell
\end{array}\right) k \ell\left(\frac{\Delta^{2} n}{2 m-6 \ell_{0}}\right)^{k+\ell} \leq 2 n^{1 / 2} \ell_{0}^{2} \log ^{3 \ell_{0}} n .
$$

(We remind the reader that it is possible to remove the $O\left(n^{1 / 2}\right)$ factor here. This would be worth doing if we could reduce $\varepsilon$ to $O(\log \log n / \log n)$. This should become apparant in the proof of Lemma 6.8, equation (31)).

Part (b) follows from the Markov inequality.

(c) For a fixed $s$, the probability such a set exists can be bounded by

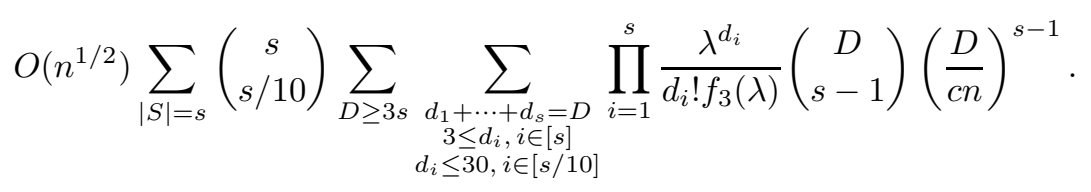

Explanation: We choose a set $S$ and we let the degrees in $S$ be $d_{1}, d_{2}, \ldots, d_{s}$ where $D$ is the total degree. Since the induced subgraph is connected, it must contain a spanning tree. We weaken this to it must contain $s-1$ edges. $\left(\begin{array}{c}D \\ s-1\end{array}\right)$ enumerates the lower numbered point of the edges and then $D^{s-1}$ enumerates the other possible endpoints and then $\left(\frac{1}{c n-2 s}\right)^{s-1}=\frac{1+o(1)}{(c n)^{s-1}}$ bounds the probability the selected pairs exist.

We bound this by

$$
\begin{aligned}
& O\left(n^{1 / 2}\right)\left(\begin{array}{c}
n \\
s
\end{array}\right)\left(\begin{array}{c}
s \\
s / 10
\end{array}\right) \sum_{D \geq 3 s}\left(\begin{array}{c}
D \\
s
\end{array}\right)\left(\frac{D}{c n}\right)^{s-1} f_{3}(\lambda)^{-s}\left[x^{D}\right]\left(\sum_{i=3}^{30} \frac{\lambda^{i} x^{i}}{i !}\right)^{s / 10} f_{3}(\lambda x)^{9 s / 10} \\
& \leq O\left(n^{3 / 2}\right)\left(\frac{e}{s}\right)^{s}(10 e)^{s / 10} \sum_{D \geq 3 s}\left(\frac{D e}{s}\right)^{s}\left(\frac{D}{c}\right)^{s-1} \frac{1}{f_{3}(\lambda)^{s}(1+\xi)^{D}}\left(\sum_{i=3}^{30} \frac{\lambda^{i}(1+\xi)^{i}}{i !}\right)^{s / 10} f_{3}(\lambda(1+\xi))^{9 s / 10}
\end{aligned}
$$

for any positive $\xi$.

Now if $\lambda$ is large then $f_{3}(\lambda) \geq e^{\lambda} / 2$. Also, $f_{3}(\lambda(1+\xi)) \leq e^{\lambda(1+\xi)}$. Furthermore,

$$
\sum_{i=3}^{30} \frac{\lambda^{i}(1+\xi)^{i}}{i !} \leq 2 \frac{\lambda^{30} e^{30 \xi}}{30 !} \leq 2\left(\frac{\lambda e^{1+\xi}}{30}\right)^{30}
$$

We will take $\xi$ to be small but fixed. Then the bound becomes

$$
O\left(n^{3 / 2}\right)(10 e)^{s / 10}\left(\frac{e^{2}}{c s^{2}}\right)^{s} \frac{2^{s / 10} e^{9 \lambda \xi s / 10}}{e^{\lambda s / 10}}\left(2\left(\frac{\lambda e^{1+\xi}}{30}\right)^{30}\right)^{s / 10} \sum_{D \geq 3 s} \frac{D^{2 s-1}}{(1+\xi)^{D}}
$$


We observe that if $u_{D}=\frac{D^{2 s}}{(1+\xi)^{D}}$ then $u_{D+1} / u_{D} \leq \frac{1+3 / D}{1+\xi}$ for $D \geq 9$. So,

$$
\sum_{D \geq 3 s} \frac{D^{2 s-1}}{(1+\xi)^{D}} \leq(3 s)^{2 s} \sum_{D \geq 3 s} \frac{1}{(1+\xi)^{D}} \prod_{i=3 s}^{D} \frac{i+3}{i} \leq(3 s)^{2 s} \sum_{D \geq 3 s} \frac{(D+3)^{3}}{(3 s)^{3}(1+\xi)^{D}} \leq \frac{(3 s)^{2 s}}{\xi} .
$$

For the last inequality we use the fact that $s$ is large and then $D^{3} \ll(1+\xi)^{D}$.

Continuing, we get a bound of

$$
\begin{aligned}
& \leq O\left(n^{3 / 2}\right)(10 e)^{s / 10}\left(\frac{e^{2}}{c s^{2}}\right)^{s} \frac{2^{s / 10} e^{9 \lambda \xi s / 10}}{e^{\lambda s / 10}}\left(2\left(\frac{\lambda e^{1+\xi}}{30}\right)^{30}\right)^{s / 10}(3 s)^{2 s} \\
& =O\left(n^{3 / 2}\right)\left(\frac{9(40 e)^{1 / 10} e^{2}}{c e^{\lambda(1-9 \xi) / 10}}\left(\frac{\lambda e^{1+\xi}}{30}\right)^{5}\right)^{s} \\
& =o(1)
\end{aligned}
$$

if we take $\xi=1 / 10$ and $c$ and hence $\lambda$ sufficiently large.

\section{Finding a Hamilton cycle}

We assume that we have a path $P$ with endpoints $a, b$ and we do rotations with $a$ as the fixed endpoint to try to find an extension. In the next section we show that if no extensions are found, then w.h.p. we create sufficient endpoints other than $b$ on paths of length equal to $P$. Throughout this description, we will assume that no extension is found i.e. all neighbors of endpoints turn out to be vertices of $P$. We associate the search with something similar to an alternating tree of matching theory.

\subsection{Tree Growth}

In this section we describe our search for a longer path than $P$ using EXTEND-ROTATE in terms of growing a tree structure, where each vertex determines a new long path. We expose what happens w.h.p. if we fail to find an extension. Let $A_{0}=\{b\}$ and let $B_{0}$ be the set of neighbors of $b$ on $P$, excluding $b$ 's path neighbor. We now define the sets $A_{i}, B_{i}, i=1, \ldots$, and $C_{i}=\bigcup_{j \leq i}\left(A_{j} \cup B_{j}\right)$. Here every vertex $v$ in $A_{i}$ will be the endpoint of a path of the same length as $P$. It will be obtained from $P$ by exactly $i$ rotations with $a$ as the fixed endpoint. Fix $i \geq 0$ and let $A_{i}=\left\{v_{1}, v_{2}, \ldots, v_{k}\right\}$. We build $A_{i+1}, B_{i+1}$ by examining $v_{1}, v_{2}, \ldots, v_{k}$ in this order. Initially $A_{i+1}=B_{i+1}=\emptyset$ and we will add vertices as we process the vertices of $A_{i}$. Fix $v=v_{j}$. We have a path $P_{v}$ with endpoints $a, v$. We consider two cases:

Case 1: $\left|C_{i}\right| \leq L_{0}=\frac{1}{20} \log _{c} n$.

Let $N_{v}=\left\{u_{1}, u_{2}, \ldots, u_{d}\right\}$ be the neighbors of $v$, excluding its neighbor on $P_{v}$. We also exclude from $N_{v}$ those neighbors already in $B_{i+1}$ (as defined so far). Let $w_{l}$ be the neighbor of $u_{l}$ on $P_{v}$ that lies between $u_{l}$ and $v$ for $l=1,2, \ldots, d$. Let $N_{v}^{\prime}=\left\{w_{1}, w_{2}, \ldots, w_{d}\right\}$. We exclude from $N_{v}^{\prime}$ those vertices already in $A_{i+1}$ (as defined so far). We add $N_{v}$ to $B_{i+1}$ and $N_{v}^{\prime}$ to $A_{i+1}$ and we add edges $\left(v, u_{j}\right)$ and $\left(u_{j}, w_{j}\right)$ to $T$. The edge $\left(u_{j}, w_{j}\right)$ will be called a lost edge. Furthermore, we define $P_{w_{j}}=P_{v}+\left(v, u_{j}\right)-\left(u_{j}, w_{j}\right)$ and observe that $P_{w_{j}}$ has endpoints $b, w_{j}$.

Case 2: $\left|C_{i}\right|>L_{0}$.

Now let $N_{v}=\left\{u_{1}, u_{2}, \ldots, u_{d}\right\}$ be its neighbors as above. We now exclude from $N_{v}$ those neighbors already in $C_{i+1}$ (as defined so far) as well as those $u_{j}$ for which $w_{j} \in C_{i+1}$. We define $N_{v}^{\prime}$ and update $A_{i+1}, B_{i+1}, T$ with this restricted $N_{v}$. 
We define the subgraph $T=T(P, b, k)$ as follows: It has vertex set $C_{k}$ plus the edges of the form $\left(v, u_{j}\right)$ and $\left(u_{j}, w_{j}\right)$ used above. $T$ suggests a tree. It is usually a tree, but in rare cases it may be unicyclic. This follows from Lemma 5.1. When this happens, some $v \in A_{i}$ (Case 1) has a neighbor in $B_{j}, j \leq i$.

We see from this that w.h.p. $T$ defined prior to the lemma has at most one cycle. By construction, cycles of $T$ are contained in the first $i_{0}$ levels. If there are two cycles inside the first $i_{0}$ levels then there is a set $S$ (consisting of the two cycles plus a path joining them) with at most $4 i_{0}$ vertices and at least $|S|+1$ edges.

We argue next that w.h.p. $T$ can be assumed to grow to a certain size and we can control its rate of growth.

Lemma 6.1. Let $\beta$ be some small fixed positive constant. If $c$ is sufficiently large, then for all paths $P$ and endpoints b such that extension does not occur, w.h.p.

(a) There exists $k$ such that $\left|C_{k}\right| \geq L_{0}=\frac{1}{15} \log _{c} n$.

(b) If $L_{0} \leq\left|C_{k}\right| \leq n^{.6}$ then $\left|A_{k+1}\right| \in\left[2(1-\beta) c\left|C_{k}\right|, 2(1+\beta) c\left|C_{k}\right|\right]$, even if only punctual edges are used once $\left|A_{k}\right|$ reaches size at least $n^{\varepsilon}$.

(c) There exists $k_{0}=O\left(\log _{c} n\right)$ such that $\left|A_{k_{0}}\right| \in\left[(2 c(1+\beta))^{-1} n^{1 / 2+5 \varepsilon}, 2 c(1+\beta) n^{1 / 2+5 \varepsilon}\right]$.

(d) Let $k_{1}=k_{0}-\ell_{0}$ where $\ell_{0}=2 \log \log n$ and let $x \in A_{k_{1}}$. Let $S$ be the set of descendants of $x$ in $A_{k_{0}}$ and let $s=|S|$. Let $S_{0}=\{y \in S: d(y) \geq 30\}$ and let $s_{0}=\left|S_{0}\right|$. Then, where $W_{1}$ is as in Lemma 5.1,

(i) $x \notin W_{1}$ and $s \geq(2 c(1-\beta))^{k_{0}-k_{1}} / 4$ implies that $s_{0} \geq 99 s / 100$.

(ii) $s \leq(2 c(1+\beta))^{k_{0}-k_{1}} \log n$.

Proof (a) Lemma 2.1 of [12] proves the following: Suppose that $S$ is the set of endpoints that can be produced by considering all possible sequences of rotations starting with some fixed path $P$ and keeping one endpoint fixed. Let $T$ be the set of external neighbors of $S$. Here $S \cap T=\emptyset$. Then $|T| \leq 2|S|$ and $S \cup T$ conmtains strictly more than $|S \cup T|$ edges. The assumption here is that the graph involved has minimum degree at least three. It follows from Lemma 5.1 that $|S| \geq \frac{1}{15} \log _{c} n$. As a final check, if $\left|C_{k}\right|$ never reached $L_{0}$ then it would have explored all possible sets of endpoints i.e. the breadth first search is no restriction.

(b) If the condition in (b) fails then the following structure appears: Let $\delta=1$ if $T$ is not a tree and 0 otherwise. Let $\operatorname{EVEN}(T)=\bigcup_{i=0}^{k} A_{i}$ and $O D D(T)=\bigcup_{i=0}^{k} B_{i}$ where $k$ is the number of iterations involved in the construction of $T$. Then with $|E V E N(T)|=l$ and $|N(E V E N(T))|=r$ we have (i) $2(l-1)+\delta$ edges of $T$ connecting $E V E N(T)$ to $\operatorname{Odd}(T)$ (ii) $r-l+1$ edges connecting $E V E N(T)$ to $N(E V E N(T)) \backslash \operatorname{Odd}(T)$ and (iii) none of the $l(n-r-l)$ edges between $E V E N(T)$ and $V \backslash N(E V E N(T))$ are present.

Assume first that $T$ is actually a tree and that $l \leq n^{\varepsilon}$ so that the edges of $T$ need not be punctual.

Given the vertices of $T$ and $N(E V E N(T))$, the probability of the existence of a $T$ with $L_{0} \leq l \leq n^{6}$ and $r \leq(1-\beta) c l$ can be bounded by

$$
O(\sqrt{n})\left(\frac{1}{2 m-2(l+r)}\right)^{l+r-1} \sum_{\substack{d_{i} \geq 3, i \in[r+l-1] \\ \sum_{i=1}^{l} d_{i}=r+l-1}}\left(\prod_{i=1}^{l} \frac{\lambda^{d_{i}} d_{i} !}{d_{i} ! f_{3}(\lambda)} \prod_{i=l+1}^{2 l-1} \frac{\lambda^{d_{i}} d_{i}\left(d_{i}-1\right)}{d_{i} ! f_{3}(\lambda)} \prod_{i=2 l}^{r+l-1} \frac{\lambda^{d_{i}} d_{i}}{d_{i} ! f_{3}(\lambda)}\right)
$$

Explanation: The probability that an edge exists between vertices $u$ and $v$ of degrees $d_{u}$ and $d_{v}$, given the existence of other edges in $T$, is at most $\frac{d_{u}^{\prime} d_{v}^{\prime}}{2 m-2(l+r)+3}$ where $d_{u}^{\prime}=d_{u}$ less the number of edges already assumed to be incident with $u$. Hence, given the degree sequence, the probability that $T$ exists is at most

$$
\left(\frac{1}{2 m-2(l+r)}\right)^{l+r-1} \prod_{i=1}^{l} d_{i} ! \prod_{i=l+1}^{2 l-1} d_{i}\left(d_{i}-1\right) \prod_{i=2 l}^{r+l-1} d_{i}
$$


(We dropped the +3 in $2 m-2(l+r)+3)$.

Here the first product corresponds to $E V E N(T)$, the second product corresponds to $O D D(T)$ and the final product corresponds to neighbours of $T$ (not in $T$ ).

We will implicitly use the fact that if $c$ is sufficiently large, then so is $\lambda$.

We now simplify the expression (8) obtained for the probability to

$$
\begin{aligned}
& O(\sqrt{n})\left(\frac{1}{2 m-2(l+r)}\right)^{l+r-1} \times \\
& \frac{\lambda^{2 r+2 l-3}}{f_{3}(\lambda)^{r+l-1}} \sum_{\sum_{i=1}^{l} d_{i=r+l-1}}\left(\sum_{d_{i} \geq 3, i \in[r+l-1]} \prod_{i=l+1}^{2 l-1} \frac{\lambda^{d_{i}-2}}{\left(d_{i}-2\right) !} \prod_{i=2 l}^{r+l-1} \frac{\lambda^{d_{i}-1}}{\left(d_{i}-1\right) !}\right) \\
& \leq O(\sqrt{n})\left(\frac{1}{2 m-2(l+r)}\right)^{l+r-1} \times \\
& \left.\frac{\lambda^{2 r+2 l-3}}{f_{3}(\lambda)^{r+l-1}} \sum_{\sum_{i=1}^{l} d_{i}=r+l-1}\left(\prod_{i=l+1}^{2 l-1} \sum_{d_{i} \geq 3} \frac{\lambda^{d_{i}-2}}{\left(d_{i}-2\right) !}\right)\left(\prod_{i=2 l}^{r+l-1} \sum_{d_{i} \geq 3} \frac{\lambda^{d_{i}-1}}{\left(d_{i}-1\right) !}\right)\right) \\
& \leq O(\sqrt{n})\left(\frac{1}{2 m-2(l+r)}\right)^{l+r-1} \frac{\lambda^{2 r+2 l-3}}{f_{3}(\lambda)^{r+l-1}}\left(\begin{array}{l}
r \\
l
\end{array}\right) f_{1}(\lambda)^{l-1} f_{2}(\lambda)^{r-l} \\
& \leq O(\sqrt{n})\left(\frac{1}{2 m-2(l+r)}\right)^{l+r-1} \frac{\lambda^{2 r+2 l-3}}{f_{3}(\lambda)^{r+l-1}}\left(\frac{e r}{l}\right)^{l} f_{1}(\lambda)^{l-1} f_{2}(\lambda)^{r-l} \\
& =O(\sqrt{n})\left(\frac{1}{2 m-2(l+r)}\right)^{l+r-1}\left(\frac{e r}{l}\right)^{l} \frac{(2 c \lambda)^{r}}{\lambda f_{2}(\lambda)}\left(\frac{2 c \lambda f_{1}(\lambda)}{f_{2}(\lambda)^{2}}\right)^{l-1}
\end{aligned}
$$

using $\frac{\lambda f_{2}(\lambda)}{f_{3}(\lambda)}=2 c$

$$
\begin{aligned}
& \leq O(\sqrt{n})\left(\frac{1}{2 m-2(l+r)}\right)^{l+r-1} \frac{2 e(1-\beta) c(2 c \lambda)^{r}}{\lambda f_{2}(\lambda)}\left(\frac{4(1-\beta) e c^{2} \lambda f_{1}(\lambda)}{f_{2}(\lambda)^{2}}\right)^{l-1} \text { using } r / l \leq 2(1-\beta) c \\
& \leq O(\sqrt{n})\left(\frac{1}{2 m-2(l+r)}\right)^{l+r-1} \frac{2 e(1-\beta) c(2 c \lambda)^{r}}{\lambda f_{2}(\lambda)}\left(\frac{4\left(1-\beta^{2}\right) e c^{2} \lambda}{f_{2}(\lambda)}\right)^{l-1} \text { using } \frac{f_{1}(\lambda)}{f_{2}(\lambda)}<1+\beta \\
& \leq O(\sqrt{n})\left(\frac{1}{2 c n}\right)^{l+r-1} e^{3(l+r)^{2} / 2 c n}(2 c \lambda)^{r}\left(\frac{4 e c^{2} \lambda}{f_{2}(\lambda)}\right)^{l-1} \text { using } m=c n \\
& =O(\sqrt{n})\left(\frac{1}{n}\right)^{l+r-1} e^{o(l)} \lambda^{r}\left(\frac{2 e c \lambda}{f_{2}(\lambda)}\right)^{l-1}
\end{aligned}
$$

since $r=O(l)$.

We now count the number of such configurations. We begin by choosing $\operatorname{EVEN}(T)$ and the root vertex of the tree in at most $n\left(\begin{array}{c}n \\ l-1\end{array}\right)$ ways. We make the following observation about $T$. The contraction of the lost edges of the tree yields a unique tree on $l$ vertices. We note, by Cayley's formula, that the number of trees that could be formed using $l$ vertices is $l^{l-2}$. Reversing this contraction, we now choose the sequence of $l$ vertices, $\operatorname{Odd}(T)$, that connect up vertices in $\operatorname{EVEN}(T)$ in $(n-l)(n-l-1) \ldots(n-2 l+1)=(n-l)_{l}$ ways. We pick the remaining $r-l$ vertices from the remaining $n-2 l$ vertices in $\left(\begin{array}{c}n-2 l \\ r-l\end{array}\right)$ ways. These $r-l$ vertices can connect to any of $E V E N(T)$ in $l^{r-l}$ ways. Hence, the total number of choices for $T$ is at most

$$
\left(\begin{array}{c}
n \\
l
\end{array}\right) l^{l-2}(n-l)_{l}\left(\begin{array}{c}
n-2 l \\
r-l
\end{array}\right) l^{r-l} \leq n^{r+l} e^{r}\left(\frac{l}{r-l}\right)^{r-l} .
$$


Combining the bounds for probability and choices of $T$, we get an upper bound of

$$
n^{r+l} e^{r}\left(\frac{l}{r-l}\right)^{r-l} O(\sqrt{n})\left(\frac{1}{n}\right)^{l+r-1} e^{o(l)} \lambda^{r}\left(\frac{2 e c \lambda}{f_{2}(\lambda)}\right)^{l-1} \leq O\left(n^{3 / 2}\right) \cdot\left(\frac{e \lambda l}{r-l}\right)^{r-l}\left(\frac{4 e^{2+o(1)} c \lambda^{2}}{f_{2}(\lambda)}\right)^{l-1}
$$

The expression $\left(\frac{e \lambda l}{x}\right)^{x}$ is maximized at $x=\lambda l$. Our assumptions imply that $r \leq 2(1-\beta) c l<\lambda l$. Hence, we have the bound

$$
\begin{aligned}
& O\left(n^{3 / 2}\right) \cdot\left(\frac{e \lambda l}{2(1-\beta) c l}\right)^{2(1-\beta) c l}\left(\frac{30 c \lambda^{2}}{f_{2}(\lambda)}\right)^{l} \\
& \leq O\left(n^{3 / 2}\right) \cdot\left(\left(\frac{e}{1-\beta}\right)^{2(1-\beta) c} \cdot \frac{120 c^{3}}{f_{2}(\lambda)}\right)^{l} \quad \text { using } \lambda<2 c \\
& \leq O\left(n^{3 / 2}\right) \cdot e^{-\beta c l}
\end{aligned}
$$

using

$$
f_{2}(\lambda)>\frac{120 c^{3} e^{(2-\beta) c}}{(1-\beta)^{2(1-\beta) c}}
$$

for $c$ sufficently large.

We sum $O\left(n^{3 / 2}\right) \cdot e^{-\beta c l}$ over all $r$ and $l$ with $L_{0} \leq l \leq n^{0.6}$ and $l \leq r \leq(1-\beta) c l$ and we get the probability to be at most

$$
O\left(n^{7 / 2}\right) e^{-\beta c L_{0}}=o(1)
$$

for $c$ sufficiently large.

We now consider the probability of the existence of a $T$ having $L_{0} \leq l \leq n^{0.6}$ and $r \geq 2(1+\beta) c l$. Note that we can assume $r \leq l \Delta \leq l \log n$ here.

The bound (10) remains valid. Replacing $r$ by $r+1$ multiplies this by a factor $O\left(c n^{-1} e^{l / r}\right)$ and so for this bound we can just assume that $r=2(1+\beta)$ cl. This changes the $1-\beta$ in (11) to $1+\beta$ and we replace (12) by

$$
O(\sqrt{n})\left(\frac{1}{n}\right)^{l+r-1} e^{o(l)} \lambda^{r}\left(\frac{2 e c(1+\beta)^{2} \lambda}{f_{2}(\lambda)}\right)^{l-1} .
$$

We re-use (13) and replace (14) by

$$
O\left(n^{3 / 2}\right) \cdot\left(\frac{e \lambda l}{r-l}\right)^{r-l}\left(\frac{4 e^{2+o(1)}(1+\beta)^{2} c \lambda^{2}}{f_{2}(\lambda)}\right)^{l-1} .
$$

Our assumptions imply that $r=2(1+\beta) c l>\lambda l$. Hence, we have the bound

$$
\begin{aligned}
& O\left(n^{3 / 2}\right) \cdot\left(\frac{e \lambda l}{2(1+\beta) c l}\right)^{2(1+\beta) c l}\left(\frac{30(1+\beta)^{2} c \lambda^{2}}{f_{2}(\lambda)}\right)^{l} \\
& \leq O\left(n^{3 / 2}\right) \cdot\left(\left(\frac{e}{2(1+\beta)}\right)^{2(1+\beta) c} \cdot \frac{120(1+\beta)^{2} c^{3}}{f_{2}(\lambda)}\right)^{l} \quad \text { using } \lambda<c \\
& \leq O\left(n^{3 / 2}\right) \cdot e^{-\beta c l}
\end{aligned}
$$

using

$$
f_{2}(\lambda)>\frac{120(1+\beta)^{2} c^{3} e^{(2+4 \beta) c}}{(2(1+\beta))^{2(1+\beta) c}}
$$

for $c$ sufficiently large. 
We sum $O\left(n^{3 / 2}\right) \cdot e^{-\beta c l}$ over all $r$ and $l$ with $L_{0} \leq l \leq n^{0.6}$ and $r \geq 2(1+\beta) c l$ and we get the probability to be at most

$$
O\left(n^{7 / 2}\right) e^{-\beta c L_{0}}=o(1)
$$

for $c$ sufficiently large.

We next consider the case where $l \geq n^{\varepsilon}$ and we can only use $O\left(n^{\varepsilon}\right)$ late edges. We will use (13), which is still an upper bound and only modify (8). Let

$$
b\left(d, d^{\prime}, 1-\alpha\right)=\left(\begin{array}{c}
d \\
d^{\prime}
\end{array}\right) \frac{((1-\alpha) m)_{d^{\prime}}(\alpha m)_{d-d^{\prime}}}{(m)_{d}}=\left(\begin{array}{c}
d \\
d^{\prime}
\end{array}\right)(1-\alpha)^{d^{\prime}} \alpha^{d-d^{\prime}}\left(1+O\left(\frac{\log ^{2} n}{n}\right)\right)
$$

for $d \leq \Delta \leq \log n$.

We replace (8) by

$$
\begin{aligned}
& O(\sqrt{n})\left(\frac{1}{2 m-2(l+r)}\right)^{l+r-1} \times \\
& \sum_{d_{i} \geq 3} \sum_{\substack{d_{i}^{\prime} \leq d_{i}, i \in[l] \\
\sum_{i=1}^{l} d_{i}^{\prime}=r+l-1}}\left(\prod_{i=1}^{l} \frac{\lambda^{d_{i}} d_{i}^{\prime} !}{d_{i} ! f_{3}(\lambda)} b\left(d_{i}, d_{i}^{\prime}, 1-\alpha\right) \prod_{i=l+1}^{2 l-1} \frac{\lambda^{d_{i}} d_{i}\left(d_{i}-1\right)}{d_{i} ! f_{3}(\lambda)} \prod_{i=2 l}^{r+l-1} \frac{\lambda^{d_{i}} d_{i}}{d_{i} ! f_{3}(\lambda)}(1-\alpha)\right) \\
& =O(\sqrt{n})\left(\frac{1}{2 m-2(l+r)}\right)^{l+r-1} \times \frac{\lambda^{2 r+2 l-3}(1-\alpha)^{2 r-1}}{f_{3}(\lambda)^{r+l-1}} \times \\
& \sum_{d_{i} \geq 3} \sum_{\substack{\sum_{i=1}^{l} d_{i}^{\prime} \leq d_{i} \\
d_{i}^{\prime}=r+l-1}} \prod_{i=1}^{l} \frac{(\lambda \alpha)^{d_{i}-d_{i}^{\prime}}}{\left(d_{i}-d_{i}^{\prime}\right) !}\left(\left(\prod_{i=l+1}^{2 l-1} \sum_{d_{i} \geq 3} \frac{\lambda^{d_{i}-2}}{\left(d_{i}-2\right) !}\right)\left(\prod_{i=2 l}^{r+l-1} \sum_{d_{i} \geq 3} \frac{\lambda^{d_{i}-1}}{\left(d_{i}-1\right) !}\right)\right) \\
& \leq O(\sqrt{n})\left(\frac{1}{2 m-2(l+r)}\right)^{l+r-1} \times \frac{\lambda^{2 r+2 l-3}(1-\alpha)^{2 r-1}}{f_{3}(\lambda)^{r+l-1}} \times \\
& \sum_{\substack{d_{i}^{\prime} \geq 1 \\
d_{i}^{\prime}=r+l-1}}\left(\sum_{\kappa \geq 0} \frac{(\lambda \alpha)^{k}}{k !}\right)^{l}\left(\prod_{i=l}^{2 l-1} \sum_{d_{i} \geq 3} \frac{\lambda^{d_{i}-2}}{\left(d_{i}-2\right) !}\right)\left(\prod_{i=2 l}^{r+l-1} \sum_{d_{i} \geq 3} \frac{\lambda^{d_{i}-1}}{\left(d_{i}-1\right) !}\right) \\
& \leq O(\sqrt{n})\left(\frac{1}{2 m-2(l+r)}\right)^{l+r-1} \times \frac{\lambda^{2 r+2 l-3}(1-\alpha)^{2 r}}{f_{3}(\lambda)^{r+l-1}} \times\left(\begin{array}{l}
r \\
l
\end{array}\right) e^{\lambda \alpha l} f_{1}(\lambda)^{l-1} f_{2}(\lambda)^{r-l} .
\end{aligned}
$$

Explanation for (19): $d_{i}$ is the degree of vertex $i$ and for $i \in[l], d_{i}^{\prime}$ is the "early" degree. The factor $b\left(d_{i}, d_{i}^{\prime}, 1-\alpha\right)$ is the probability that $i$ has $d_{i}^{\prime}$ neighbors.

Observe now that the expression in (20) is precisely

$$
e^{\lambda \alpha l}(1-\alpha)^{2 r} \leq e^{\alpha(\lambda l-2 r)}
$$

times the expression in (9). It follows that the probability bound (15) can be replaced by

$$
O\left(n^{1 / 2}\right) \cdot e^{-\beta c l} \cdot e^{\alpha(\lambda l-2 r)} \leq O\left(n^{1 / 2}\right) \cdot e^{-\beta c l / 2} .
$$

We sum this over $l, r$ to get the required conclusion.

The case $r \geq 2(1+\beta) c l$ for $l \leq n^{\varepsilon}$, using only punctual edges follows a fortiori from the previous analysis.

We finally consider the case where $T$ is not a tree. When this happens, it will be because of a unique (Lemma 5.1) edge introduced in Case 1. We can be handle this by multiplying our final estimates by 
$O\left(L_{0}^{2} n^{-1} \log ^{2} n\right)$. The factor $O\left(L_{0}^{2}\right)$ accounts for choosing a pair of vertices in $T$ in Case 1 and $O\left(n^{-1} \log ^{2} n\right)$ bounds the probability of the existence of this edge, given previous edges.

Part (c) follows from (b).

(d) If we consider the growth of the sub-tree emanating from $x$ then we can argue that it grows as fast as described in (a) and (b). We just have to deal with the edges pointing into the part of $T$ that has already been constructed. We can argue as for (19) with $\alpha=o(1)$, since the chances of choosing an endpoint in $T$ is $o(1)$ at each point.

If $x \notin W_{1}$ then the descendants $D_{i}$ of $x$ at levels $k_{0}+i$ grow at a rate of at least two (i.e. $\left|D_{i+1}\right| \geq 2\left|D_{i}\right|$ ) for $O(\log \log n)$ steps until $\left|D_{i}\right| \gg \log n$ and after this will grow at a rate of al least $2 c(1-\beta)$. In which case the leaves of $T_{x}$, the sub-tree of $T$ rooted at $x$, will constitute a fraction $1-O(1 / c)$ of the vertices of $T_{x}$. The result now follows from Lemma 5.1 (c).

If $x \in W_{1}$ then $\left|D_{i}\right|$ grows at a rate of at most $2 c(1+\beta)$ once it has reached size $\log n$.

Remark 6.1. It follows from this lemma that only $O\left(n^{1 / 2+O(\varepsilon)}\right)$ tardy edges are needed to build all of the instances of $A_{k_{0}}$ needed by EXTEND-ROTATE. If one looks at Section 4.3.1 of [8] one sees, in conjunction with equation (1) of that paper that the total running time of Step $3 b$ of this paper is $O\left(n^{.995+o(1)}\right)$ and so we can use this as a bound on the number of punctual edges examined by Step 3b. We can drastically reduce this in the same way we did for building the trees in EXTEND-ROTATE, but since we are only claiming our result for $c$ sufficiently large and $\varepsilon \ll .005$, this is not necessary, since there will w.h.p. be $\Omega\left(n^{1-2 \varepsilon}\right)$ tardy $R_{0}: \Lambda_{0}$ edges, see Lemma 6.5 below. In other words, almost all of the tardy $R_{0}: \Lambda_{0}$ edges are not using for tree building.

The above lemma shows that $A_{k}$ can be relied on to get large. Unfortunately, we need to do some more analysis because we do not have full independence, having run 2GREEDY. Normally, one would only have to show that $\operatorname{END}(a)$ is large for all relevant vertices $a$ and this would be enough to show the existence w.h.p. of an edge joining $a$ to $b \in \operatorname{END}(a)$ for some $a, b$. We will have to restrict our attention to the case where $a \in R_{0}$ and $b \in \Lambda_{0}$, see Remark 4.1. So first of all we will show that w.h.p. there are many $a \in R_{0}$, see Lemma 6.8. For this we need to show that every path we come across contains many consecutive triples $u, v, w \in R_{0}$. In which case, an inserted edge $(x, v)$ produces a path with an endpoint in $R_{0}$. We also need to show that w.h.p. there are many $b \in \Lambda_{0}$, see Lemma 6.7. We will also need to show that there are many edges that can be $(a, b)$, see Lemma 6.5.

For the Lemma 6.3 below we need some results from [10]. Let $\mathbf{u}=\mathbf{u}(t)$ denote $(y(t), z(t), \mu(t))$ and let $\hat{\mathbf{u}}=\hat{\mathbf{u}}(t)$ denote $(\hat{y}(t), \hat{z}(t), \hat{\mu}(t))$ where $y(t)$ etc. denotes the value of $y=|Y|, z=|Z|, \mu=\mid E(\Gamma(t) \mid$ at time $t$ and $\hat{y}(t)$ etc. denotes the deterministic value for the solution to the associated set of differential equations, summarised in equation (152) of that paper:

$$
\frac{d \hat{y}}{d t}=\hat{A}+\hat{B}-\hat{C}-1 ; \quad \frac{d \hat{z}}{d t}=2 \hat{C}-2 \hat{A}-2 \hat{B} ; \quad \frac{d \hat{\mu}}{d t}=-1-\hat{D} .
$$

where

$$
\hat{A}=\frac{\hat{y} \hat{z} \hat{\lambda}^{5} f_{0}(\hat{\lambda})}{8 \hat{\mu}^{2} f_{2}(\hat{\lambda}) f_{3}(\hat{\lambda})}, \quad \hat{B}=\frac{\hat{z}^{2} \hat{\lambda}^{4} f_{0}(\hat{\lambda})}{4 \hat{\mu}^{2} f_{2}(\hat{\lambda})^{2}}, \quad \hat{C}=\frac{\hat{y} \hat{\lambda} f_{2}(\hat{\lambda})}{2 \hat{\mu} f_{3}(\hat{\lambda})}, \quad \hat{D}=\frac{\hat{z} \hat{\lambda}^{2} f_{0}(\hat{\lambda})}{2 \hat{\mu} f_{2}(\hat{\lambda})}
$$

and $f_{j}(x)=e^{x}-\sum_{i=0}^{j-1} \frac{x^{i}}{i !}$.

Lemma 7.1 of [10] proves that $\mathbf{u}(t)$ and $\hat{\mathbf{u}}(t)$ are close w.h.p.:

Lemma 6.2.

$$
\|\mathbf{u}(t)-\hat{\mathbf{u}}(t)\|_{1} \leq n^{8 / 9}, \quad \text { for } 1 \leq t \leq \min \left\{T_{0}, \hat{T}_{0}\right\} \text { w.h.p.. }
$$

Here $T_{0}$ is a stopping time and $\hat{T}_{0}$ is a deterministic time such that w.h.p. Step 3 begins before $\min \left\{T_{0}, \hat{T}_{0}\right\}$. 
Note that $\varepsilon \ll 1 / 9$. Let

$$
i_{o}=n^{3 / 4-\varepsilon} \text { and } \rho=n^{1 / 4} .
$$

Equation (163) of [10] states that w.h.p.

$$
\left|\theta_{\xi}(\hat{\mathbf{u}}(t))-\Delta_{\xi}\right|=O\left(\rho^{-1} \log ^{2} n+\frac{\|\mathbf{u}(t)-\hat{\mathbf{u}}(t)\|_{1}}{n}\right) \text { for } \xi=a, b, c, 2
$$

Here

$$
\theta_{a}=0, \theta_{b}=\hat{A} \theta_{c}=\hat{A}+\hat{B} \text { and } \theta_{2}=1-\theta_{a}-\theta_{b}-\theta_{c}
$$

and $\Delta_{\xi}$ is the proportion of steps in $[t, t+\rho]$ that are Step $1 \xi$ or Step 2, if $\xi=2$.

Now if $\hat{z}=o(n) \hat{\mu}=\Omega(n)$ and $\hat{\lambda}=\Omega(1)$ then we have from (22) that $\hat{A}, \hat{B}=O(\hat{z} / n)$ and that $\theta_{b}=$ $O(\hat{z} / n), \theta_{c}=O(\hat{z} / n), \theta_{2}=2-o(1)$. Then from (21) we see that $\hat{z}$ grows at the rate $2-o(1)$ per time step, so long as $t=o(n)$ and hence $\hat{z}=o(n)$.

It is shown in [10] that if $c \geq 10$ then w.h.p. $\hat{\lambda}=\Omega(1)$ up until the (random) time when Step 3 begins. See equation (190) of that paper. Furthermore, it follows from Lemma 6.2 that w.h.p.

$\mathbf{X} 1$ If $t=\gamma n^{1-\varepsilon}$ for some constant $\gamma$ then w.h.p. $z(t) \sim 2 t$.

X2 If $t=\gamma n^{1-\varepsilon}$ for some constant $\gamma$ then w.h.p. there will be $O\left(n^{1-2 \varepsilon}\right)$ instances of Step 1 in $[0, t]$.

X3 $\lambda=\Omega(1)$ up until the start of Step 3.

Lemma 6.3. W.h.p., all the paths in Steps 1 and 2 of EXTEND-ROTATE contain at least $n_{0}=\Omega\left(n^{1-4 \varepsilon} / \log n\right)$ pairs of consecutive edges $(u, v),(v, w)$ such that $u, v, w \in R_{0}$.

Proof First consider the steps in the range $\left[0, i_{0} \rho / 4\right]$. It follows from $\mathbf{X} \mathbf{1}$ that at the end of this period, there will w.h.p. be at least $i_{0} \rho / 3$ vertices in $Z$. Consider the edge $(v, w)$ of Step 2 at some time in $\left[0, i_{0} \rho / 4\right]$. The probability that $w \in Z$ is certainly $\Omega\left(n^{-\varepsilon}\right)$ and the probability it has a punctual $Z$-witness is $1-\alpha-o(1)$. This holds regardless of the previous history, once we condition on an event that happens w.h.p.

The probability that $w \in Z$ is certainly $O\left(n^{-\varepsilon} \Delta\right)=O\left(n^{-\varepsilon} \log n\right)$. This implies that the number of times we create a component of $M$ containing more than two vertices is $O\left(n^{1-2 \varepsilon} \log n\right)$. Thus almost all components of $M$ at the end of the period [0.i $\left.i_{0} \rho / 4\right]$ consist of isolated edges. Let us assume then that there are at least $A_{1} n^{1-\varepsilon}$ such edges where in the following $A_{1}, A_{2}, \ldots$, are positve constants. Let $S_{1}$ denote this set of components.

Now consider the steps in the range $\left[i_{0} \rho / 4, i_{0} \rho / 2\right]$ and consider the edge $(v, w)$ of Step 2. We have $w \in V\left(S_{1}\right)$ with probability at least $A_{2} n^{-\varepsilon}$. This is because w.h.p. the total degree of $V\left(S_{1}\right)$ will be $\Omega\left(n^{1-\varepsilon}\right)$ and the total degree of $G$ is at most $2 \mathrm{cn}$. The vertex $w$ is early by construction. Also the $Z$-witness of $w$ will be punctual with probability at least $1-\alpha-O\left(n^{-\varepsilon}\right)$. We next observe that with probability at least $\left(1-\Omega\left(\frac{\Delta}{n}\right)\right)^{n^{1-\varepsilon} / 4}=1-o(1)$, this component will not be absorbed into a larger component in $\left[i_{0} \rho / 4, i_{0} \rho / 2\right]$. Thus, in expectation, at time $i_{0} \rho / 2$ there is a set of $A_{3} n^{1-2 \varepsilon}$ components of $M$ consisting of a path of length two with its middle vertex in $R_{0}$. A simple second moment calculation will show concentration around the mean, for $\left|S_{2}\right|$.

We can repeat this argument for the periods $\left[i_{0} \rho / 2,3 i_{0} \rho / 4\right],\left[3 i_{0} \rho / 4, i_{0} \rho\right]$ to argue that by time $n^{1-\varepsilon}, M$ will contain a set $S_{3}$ of at least $A_{4} n^{1-4 \varepsilon}$ components consisting of paths of length four in which the internal vertices are all in $R_{0}$.

We can argue that w.h.p. at least half of the components in $S_{3}$ will have both end vertices of degree at most $3 c$. Denote these by $S_{4}$. Indeed the number of edges incident with vertices of degree more than $3 c$ is relatively small. Indeed, the expected number of such edges is asymptotically equal to $\sum_{k \geq 3 c} \frac{k \lambda^{k}}{k ! f_{3}(\lambda)} \leq \varepsilon_{c}=(e / 3)^{3 c}$. The number of such edges is concentrated around its mean. If we assume degrees are independent and less 
than $\log n$ then we can use Hoeffding's Theorem and then correct by a factor $O\left(n^{1 / 2}\right)$ to condition on the total degree. Given this, we see that w.h.p. at least $2\left(1-\varepsilon_{c}\right)^{2} / 3$ of the components of $S_{3}$ will be created in two executions of Step 2 with the degree $v$ less than $3 c$.

Observe now that with probability at least $\left(1-\frac{6 c}{\Omega(n)}\right)^{2 c n}=\Omega(1)$ a component $C \in S_{3}$ will survive as a component of $M$ until the end of Step 2. Because $\left|S_{3}\right|$ is $O\left(n^{1-\varepsilon}\right)$, this is true regardless of which other compponents in $S_{3}$ survive. The $\Omega(n)$ in the denominator comes from the fact that w.h.p. Step 2 ends with $|Z|=\Omega(n)$. Let $S_{4}$ denote this set of components and note that w.h.p. there will be at least $A_{5} n^{1-4 \varepsilon}$ components in $S_{4}$.

Step 3 of 2 GREEDY adds a matching $M^{* *}$ that is disjoint from the edges in the contraction of $S_{3}$ to a matching. This matching is independent of $S_{3}$. This implies that w.h.p. any cycle (or possibly path) of the union of $M^{*}$ and $M^{* *}$ of length $\ell \geq n^{8 \varepsilon}$, contains at least $A_{5} \ell n^{-4 \varepsilon}$ members of $S_{4}$. Here we are using concentration of the hypergeometric distribution i.e. sampling without replacement.

In EXTEND-ROTATE we start with a path of length $\ell=\Omega(n / \log n)$ and w.h.p. every path is generated by deleting at most $O\left(\log ^{2} n\right)$ edges. This completes the proof of the lemma.

\subsection{Batches}

Let $\Gamma(t)$ denote the graph $\Gamma$ after $t$ steps of 2GREEDY. Suppose that $t_{1}<t_{2} \leq n^{1-\varepsilon}$ and that 2GREEDY applies Step 2 at times $t_{1}, t_{2}$ and Step 1 at times $t_{1}<t<t_{2}$. We consider the set of edges and vertices removed from time $t_{1}$ to time $t_{2}$, i.e. the graph $\Gamma\left(t_{1}\right) \backslash \Gamma\left(t_{2}\right)$ and call it a batch. Note that batches are connected subgraphs since each edge/vertex removed is incident to some edge that is also removed.

We also claim that each batch w.h.p. is constructed within $O\left(\log ^{2} n\right)$ steps and contains $O\left(\log ^{3} n\right)$ vertices. This follows from [10] as we now explain. Let $\zeta=y_{1}+2 y_{2}+z_{1}$. Equations (67), (68), (69) of [10] show that

$$
\mathbb{E}\left[\zeta^{\prime}-\zeta|| \mathbf{v} \mid\right]=-(1-Q)-o(1)
$$

where

$$
Q=Q(\mathbf{v})=\frac{y z}{4 \mu^{2}} \frac{\lambda^{3}}{f_{3}(\lambda)} \frac{\lambda^{2} f_{0}(\lambda)}{f_{2}(\lambda)}+\frac{z^{2}}{4 \mu^{2}} \frac{\lambda^{4} f_{0}(\lambda)}{f_{2}(\lambda)^{2}} .
$$

Lemma 6.2 of [10] shows that $1-Q=-\Omega(1)$ if $\lambda=\Omega(1)$, and $\mathbf{X 3}$ is our justification for assuming this.

Thus the expected change in $\zeta$ is $-\Omega(1)$ when $\zeta>0$. We carry out Step 2 iff $\zeta=0$. Now $\zeta$ can change by at most $O(\Delta)=O(\log n)$ and has a negative drift whenever it is positive. This implies that it must return to zero within $O\left(\log ^{2} n\right)$ steps. Another $\Delta \leq \log n$ factor will allow at most $\log n$ edges to be removed in one step. By making the hidden constant sufficently large, we can replace w.h.p. by with probability $1-O\left(n^{-10}\right)$.

\section{Lemma 6.4.}

(a) W.h.p. there are at most $n^{1-4 \varepsilon}$ vertices $v \in G$ that are within distance $\ell_{0}=2 \log \log n$ of 6 distinct batches.

(b) W.h.p. no vertex has degree more than 4 in a single batch.

\section{Proof}

(a) We bound the probability of being within distance $\ell_{0}$ of $s$ batches by

$$
\rho_{s}=\sum_{v=1}^{n}\left(\begin{array}{c}
n^{1-\varepsilon} \\
s
\end{array}\right) \prod_{i=1}^{s} \mathbb{P}\left(\operatorname{dist}\left(v, B_{i}\right) \leq \ell_{0} \mid \operatorname{dist}\left(v, B_{j}\right) \leq \ell_{0}, 1 \leq j<i\right) .
$$

Explanation: Here $\left(\begin{array}{c}n^{1-\varepsilon} \\ s\end{array}\right)$ is the number of choices for the start times of the batches $B_{1}, B_{2}, \ldots, B_{s}$. 
We claim that for each $i, v$,

$$
\mathbb{P}\left(\operatorname{dist}\left(v, B_{i}\right) \leq \ell_{0} \mid \operatorname{dist}\left(v, B_{j}\right) \leq \ell_{0}, 1 \leq j<i\right)=O\left(\frac{\log ^{2+\ell_{0}} n}{n}\right) .
$$

This gives

$$
\rho_{s} \leq \exp \left\{-(K-2-o(1))(\log \log n)^{2} s\right\} .
$$

and assuming $K \geq 13$, this implies that the expected number of vertices within distance $\ell_{0}$ of 6 batches is less than $n^{1-5 \varepsilon}$. The result now follows from the Markov inequality.

Proof of (24): Suppose that $B_{i}$ is constructed at time $t_{i}$. It is a subgraph of $\Gamma\left(t_{i}\right)$ and depends only on this graph. We argue that

$$
\mathbb{P}\left(\exists w \in B_{i}: \operatorname{dist}(v, w) \leq \ell_{0} \mid \operatorname{dist}\left(v, B_{j}\right) \leq \ell_{0}, 1 \leq j<i\right) \leq O\left(n^{-10}\right)+O\left(\frac{i \log ^{2+\ell_{0}} n}{n}\right) .
$$

Explanation: The $O\left(n^{-10}\right)$ term is the probability the batch $B_{i}$ is large. The term $O\left(\frac{i \log ^{2+\ell_{0}} n}{n}\right)$ in (25) arises as follows. We can assume that $\left|N_{\ell}(v)\right| \leq \Delta^{\ell_{0}} \leq \log ^{\ell_{0}} n$, where $N_{\ell}(v)$ is the set of vertices within distance $\ell$ of $v$. Suppose as in [3] we expose the graph $\Gamma$ at the same time that we run 2GreEDy. For us it is convenient to work within the configuration model of Bollobás [5]. Assume that we have exposed $N_{\ell}(v)$. At the start of the construction of a batch we choose a random edge of the current graph. The probability this edge lies in $N_{\ell_{0}}(w)$ is $O\left(\log ^{{ }^{\ell_{0}}} n / n\right)$. In the middle of the construction of a batch, one endpoint of an edge is known and the the other endpoint is chosen randomly from the set of configuration points associated with $\Gamma(t)$. The probability this new endpoint lies in $N_{\ell_{0}}(v)$ is also $O\left(\log ^{\ell_{0}} n / n\right)$ and there are only $O\left(\log ^{2} n\right)$ steps in the creation of a batch.

(b) The probability that vertex $v$ appears $k+3$ times in a fixed batch can be bounded above by $\left(\underset{k}{O\left(\log ^{2} n\right)}\right)\left(O\left(\frac{\Delta}{n}\right)\right)^{k}=O\left(\frac{\log ^{k+3} n}{n^{k}}\right)$. Indeed, if $v$ has degree at least 3 at any time, then the probability its degree in the current batch increases in any step is $O\left(\frac{\Delta}{n}\right)$.

We now argue that there will be a sufficient number of tardy $R_{0}: \Lambda_{0}$ edges.

Lemma 6.5. W.h.p. there will be $\Omega\left(n^{1-2 \varepsilon}\right)$ tardy $R_{0}: \Lambda_{0}$ edges.

Proof We first consider the set $F_{1}$ of tardy edges $e=(u, v)$ such that (i) $u$ appears at least twice in the first $n^{1-\varepsilon} / 10$ edges and in at least 30 other punctual edges and (ii) vertex $v$ has degree at least 30 and does not appear in the first $n^{1-\varepsilon / 2}$ edges in $\sigma$. It is straightforward to show that q.s. we have $\left|F_{1}\right|=\Theta\left(m n^{-2 \varepsilon}\right)$.

Suppose that $u$ satisfies (i). It loses at most 24 edges (Lemma 6.4(a),(b)) before the second edge incident with $u$ is chosen and then $u$ will be in $R_{0}$. This is because $u$ will be in $Z$ just before this point and will then be placed in $R$. and it will have at least six choices for a punctual $Z$-witness. We use the fact that almost all of the first $n^{1-\varepsilon}$ steps are Step 2 to see that the edges incident with $u$ occuring in the first $n^{1-\varepsilon} / 10$ steps will indeed be selected before time $n^{1-\varepsilon}$.

If $v$ satisfies (ii) and loses at most 24 edges because of Step 1 in the first $n^{1-\varepsilon}$ steps then $v$ will be in $\Lambda_{0}$. This is because it will have degree at least six in $\Gamma_{n^{1-\varepsilon}}$.

We now consider the probability that $A_{k_{0}}$ contains many vertices that lie in $\Lambda_{1}=\Lambda_{2} \cup \Lambda_{3}$ where

$$
\begin{aligned}
& \Lambda_{2}=\left\{v: v \text { appears in the first } n^{1-\varepsilon / 2} \text { edges in } \sigma\right\} \\
& \Lambda_{3}=\left\{v \notin \Lambda_{2}: v \text { loses } 24 \text { edges because of Step } 1 \text { in the first } n^{1-\varepsilon} \text { steps }\right\} .
\end{aligned}
$$

In the proof of Lemma 6.5 we used the fact that

$$
\text { if the degree of } v \text { is at least } 30 \text { and } v \notin \Lambda_{1} \text { then } v \in \Lambda_{0} .
$$


Lemma 6.6. W.h.p., every extension-rotation tree $T$ has $\left|A_{k_{0}} \cap \Lambda_{1}\right| \leq\left|A_{k_{0}}\right| / 30$.

Proof We first estimate $\left|A_{k_{0}} \cap \Lambda_{2}\right|$. We go back to (17) and estimate, for fixed $r, l$,

$$
\mathbb{P}\left(\exists T:\left|A_{k_{0}} \cap \Lambda_{2}\right| \geq l / 100\right) \leq O\left(n^{3 / 2}\right) \cdot\left(\frac{e \lambda l}{r-l}\right)^{r-l}\left(\frac{4 e^{2+o(1)}(1+\beta)^{2} c \lambda^{2}}{f_{2}(\lambda)}\right)^{l-1}\left(\begin{array}{c}
l \\
l / 200
\end{array}\right)\left(\frac{n^{1-\varepsilon / 2} \Delta}{m}\right)^{l / 200} .
$$

Explanation: We have taken the RHS of (17) and multiplied by a bound on the probability that there are at least $l / 200$ members of $E V E N(T)$ appearing in the first $n^{1-\varepsilon / 2}$ edges of $\sigma$. Note the the permutation $\sigma$ is independent of $T$ and that w.h.p. we will have $\left|A_{k_{0}}\right| \geq l / 2$. This is because for most of the time, the tree grow at a rate at least $2 c(1-\beta)$. We use (17) and not (14) because we can only assume that $r \leq 2(1+\beta) c l$.

Thus,

$$
\begin{aligned}
& \mathbb{P}\left(\exists T:\left|A_{k_{0}} \cap \Lambda_{2}\right| \geq l / 100\right) \leq \\
& \quad O\left(n^{3 / 2}\right) \cdot\left(e^{\lambda l /(r-l)} \cdot \frac{4 e^{2+o(1)}(1+\beta)^{2} c \lambda^{2}}{f_{2}(\lambda)} \cdot(200 e)^{1 / 200} \cdot\left(\frac{n^{1-\varepsilon / 2} \log n}{c n}\right)^{1 / 200}\right)^{l} \leq n^{-\varepsilon l / 300} .
\end{aligned}
$$

Now we are interested here in the case where $l=n^{1 / 2+o(1)}$ and so this is easily strong enough so that we can apply the union bound over $r, l$.

We next estimate $\left|A_{k_{0}} \cap \Lambda_{3}\right|$. We replace (27) by

$$
\begin{aligned}
& \mathbb{P}\left(\exists T:\left|A_{k_{0}} \cap \Lambda_{3}\right| \geq l / 100\right) \leq \\
& O\left(n^{3 / 2}\right) \cdot\left(\frac{e \lambda l}{r-l}\right)^{r-l}\left(\frac{4 e^{2+o(1)}(1+\beta)^{2} c \lambda^{2}}{f_{2}(\lambda)}\right)^{l-1}\left(\begin{array}{c}
l \\
l / 200
\end{array}\right)\left(\begin{array}{c}
n^{1-\varepsilon} \\
23 l / 200
\end{array}\right)\left(\frac{l \log n}{200 m}\right)^{23 l / 200} .
\end{aligned}
$$

Explanation; We have taken the RHS of (17) and multiplied by a bound on the probability that there is a set of leaves $S$ of size $l / 200$ such that at least $24 l / 200$ times during the first $n^{1-\varepsilon}$ steps the vertex $w$, the neighbor of the selected $v$, is in $S$. This is computed assuming that we have exposed the edges of $T$. Note that we have that in at most $l / 200$ times do we lose the edge of $T$ incident with $w \in S$, explaining the factor $\left(\begin{array}{c}n^{1-\varepsilon} \\ 23 l / 200\end{array}\right)$.

Equation (28) is replaced by

$$
\begin{aligned}
& \mathbb{P}\left(\exists T:\left|A_{k_{0}} \cap \Lambda_{3}\right| \geq l / 100\right) \leq \\
& O\left(n^{3 / 2} \cdot\left(e^{\lambda l /(r-l)} \cdot \frac{4 e^{2+o(1)}(1+\beta)^{2} c \lambda^{2}}{f_{2}(\lambda)} \cdot(200 e)^{1 / 200} \cdot\left(\frac{200 e n^{1-\varepsilon}}{23 l}\right)^{23 / 200} \cdot\left(\frac{l \log n}{200 c n}\right)^{23 / 200}\right)^{l} \leq n^{-\varepsilon l / 20} .\right.
\end{aligned}
$$

The next lemma puts a lower bound on $\left|\Lambda_{0} \cap A_{k_{0}}\right|$ (see Lemma 6.1).

Lemma 6.7. W.h.p. $\left|\Lambda_{0} \cap A_{k_{0}}\right| \geq\left|A_{k_{0}}\right| / 2$.

Proof Let $k_{1}=k_{0}-2 \ell_{0}$ where $\ell_{0}=2 \log \log n$. and consider $A_{k_{1}}=\left\{a_{1}, a_{2}, \ldots, a_{r}\right\}$. Note that

$$
r \geq \frac{n^{1 / 2+5 \varepsilon}}{2 c(1+\beta) \log ^{\ell_{0}} n} .
$$

Let $s_{i}$ be the number of descendents of $a_{i}$ in $A_{k_{0}}$ and let $s_{i}^{\prime}$ be the number of early descendents of $a_{i}$ in $A_{k_{0}} \cap \Lambda_{1}$. 
Let $s_{i}^{\prime \prime}$ be the number of descendents of $a_{i}$ in $A_{k_{0}}$ that have degree at most 30. We observe from Lemma 6.1(b) that

$$
\left|A_{k_{0}}\right|=\sum_{i=1}^{r} s_{i} \geq r(2 c(1-\beta))^{k_{0}-k_{1}} \geq r \log ^{\log c} n .
$$

Next let $I=\left\{i \in[r]: a_{i} \notin W_{1}\right.$ and $\left.s_{i} \geq(2 c(1-\beta))^{k_{0}-k_{1}} / 4\right\}$ (where $W_{1}$ is from Lemmas 5.1, 6.1) and observe that

$$
\sum_{i \notin I} s_{i} \leq r(2 c(1-\beta))^{k_{0}-k_{1}} / 4+n^{1 / 2} \log ^{4 \ell_{0}+1} n(2 c(1+\beta))^{k_{0}-k_{1}} \leq\left|A_{k_{0}}\right| / 3 .
$$

It follows from Lemma 6.1 (d) that

$$
s_{i}^{\prime \prime} \leq s_{i} / 5 \quad \text { for } i \in I .
$$

It follows from Lemma 6.6 that w.h.p.

$$
\sum_{i=1}^{r} s_{i}^{\prime} \leq\left|A_{k_{0}}\right| / 30
$$

Now, after using (26), we see that

$$
\left|\Lambda_{0} \cap A_{k_{0}}\right| \geq \sum_{i \in I}\left(s_{i}-s_{i}^{\prime \prime}\right)-\sum_{i=1}^{r} s_{i}^{\prime} \geq\left(\frac{4}{5} \cdot \frac{2}{3}-\frac{1}{30}\right)\left|A_{k_{0}}\right|
$$

We now consider going one iteration further and building $A_{k_{0}+1}$.

Lemma 6.8. W.h.p. $A_{k_{0}+1}$ contains at least $\Omega\left(n^{1 / 2+2 \varepsilon}\right)$ vertices of $R_{0}$. Furthermore, we can find these $R_{0}$ vertices by examining $n^{1-3 \varepsilon} \log n$ tardy $R_{0}: \Lambda_{0}$ edges.

Proof Assume from Lemmas 6.1 and 6.7 that $A_{k_{0}}$ contains at least $n_{1}=\frac{n^{1 / 2+5 \varepsilon}}{4 c^{2}(1+\beta)}$ vertices in $\Lambda_{0}$. Assume also from Lemma 6.3 that all of the paths corresponding to $A_{k_{0}}$ have $n_{0}=\Omega\left(n^{1-4 \varepsilon} / \log n\right)$ consecutive triples $u, v, w \in R_{0}$. If the middle vertex $v$ is the neighbour of an endpoint, then it yields a new endpoint of $A_{k_{0}+1}$ in $R_{0}$. Then the expected number of rotations leading to an endpoint in $R_{0}$ is at least

$$
C_{1} \times n^{1-3 \varepsilon} \log n \times \frac{n^{1 / 2+5 \varepsilon}}{3 c^{2}(1+\beta)} \times \frac{n^{1-4 \varepsilon}}{\log n} \times \frac{1}{n^{1-2 \varepsilon} \times n}=\Omega\left(n^{1 / 2+2 \varepsilon}\right)
$$

for some constant $C_{1}>0$.

We can claim a q.s. lower bound because almost all of the tardy $R_{0}: \Lambda_{0}$ edges are unconditioned, see remark 6.1 .

\section{Finishing the proof}

We have argued that we only need to do $\ell_{1}=O\left(\log ^{2} n\right)$ extensions w.h.p. The tardy $R_{0}: \Lambda_{0}$ edges are our scarce resource of residual randomness. Remark 6.1 explains that we only need to use an $o(1)$ proportion in building trees up to the $k_{0}$ th level. We will only use the result of Lemma 6.8 for growing the first extensionrotation tree of each of the $O\left(\log ^{2} n\right)$ path extensions. Lemma 6.8 tells us that we only need to use an $o(1)$ fraction of the available $R_{0}: \Lambda_{0}$ edges for producing many paths that have an $R_{0}$ endpoint.

Consider a round of EXTEND-ROTATE where we are trying to extend path $P$. We start with a path and then we construct a BFS "tree". After the first tree construction of each round, we construct $A_{k_{0}}$ and create one more level $A_{k_{0}+1}$. From Lemma 6.8, we should obtain $\Omega\left(n^{1 / 2+2 \varepsilon}\right)$ paths with early endpoints. Now we grow trees from each of these paths and try to close them using the set $E_{L}=\left\{f_{1}, f_{2}, \ldots, f_{M}\right\}$ of unused tardy 
$R_{0}: \Lambda_{0}$ edges. We can examine these edges in $\sigma$ order. The probability that the next edge $f_{i}$ fails to close a path to a cycle is $p=\Omega\left(n^{1 / 2+2 \varepsilon} \times n^{1 / 2+5 \varepsilon} \times n^{-2}\right)$. So the probability we fail is at most $\mathbb{P}\left(\operatorname{Bin}(M, p)<\ell_{1}\right)$. Now $M p=\Omega\left(n^{5 \varepsilon}\right) \gg \ell_{1}$ and so the Chernoff bounds imply that we succeed w.h.p.

As final thought, although we have proved that we can find a Hamilton cycle quickly, being very selective in our choice of edges for certain purposes, the breadth first nature of our searches imply that we can proceed in a more natural manner and use all edges available to us. In the worst-case we would have to use the designated ones.

\section{Why not $\varepsilon=O\left(\frac{\log \log n}{\log n}\right)$ ?}

In the proof of Lemma 6.1 we need to choose $\ell_{0}=2 \log \log n$ so that $2^{\ell_{0}} \gg L_{0}$ of that lemma. But then in (24) we want $n^{\varepsilon} \gg \log ^{\ell_{0}} n$. With some work we could replace the bound $\log ^{\ell_{0}} n$ by $O(c)^{\ell_{0}}$ which would allow us to take $\varepsilon=\frac{K \log \log n}{\log n}$. The catch here is that in this case we would need $K$ to grow with $c$. This is not satisfactory and so we content ourselves for now with (1).

\section{$9 \quad$ Final Remarks}

We have shown that a Hamilton cycle can w.h.p. be found in $\left.O\left(n^{1+o(1)}\right)\right)$ time. It should be possible to replace $n^{o(1)}$ by $\log ^{O(1)} n$ and we have explained the technical difiiculty in Section 8 . We think that $O\left(n \log ^{2} n\right)$ should be possible. It should also be possible to apply the ideas here to speed up the known algorithms for random regular graphs, or graphs with a fixed degree sequence.

\section{References}

[1] M. Ajtai, J. Komlós and E. Szemerédi. The first occurrence of Hamilton cycles in random graphs., Annals of Discrete Mathematics 27 (1985), 173-178.

[2] D. Angluin and L.G. Valiant, Fast probabilistic algorithms for Hamilton circuits and matchings, Journal of Computer and System Sciences 18 (1979) 155-193.

[3] J. Aronson, A.M. Frieze and B.G. Pittel, Maximum matchings in sparse random graphs: Karp-Sipser re-visited, Random Structures and Algorithms 12 (1998) 111-178.

[4] B. Bollobás, The evolution of sparse graphs, In: Graph Theory and Combinatorics, Proc. Cambridge Combin. Conf. in honour of Paul Erdős (B. Bollobás, ed.), Academic Press, 1984, pp. 35-57.

[5] B. Bollobás, A probabilistic proof of an asymptotic formula for the number of labelled regular graphs, European Journal on Combinatorics 1 (1980) 311-316.

[6] T.I.Fenner and A.M.Frieze, On the existence of hamiltonian cycles in a class of random graphs, Discrete Mathematics 45 (1983) 301-305.

[7] B.Bollobás, T.I.Fenner and A.M.Frieze, An algorithm for finding Hamilton paths and cycles in random graphs, Combinatorica 7 (1987), 327-341.

[8] P.Chebolu, A.M. Frieze and P.Melsted, Finding a Maximum Matching in a Sparse Random Graph in $O(n)$ Expected Time JACM 57, (2010) 
[9] A.M. Frieze, Finding Hamilton cycles in sparse random graphs, Journal of Combinatorial Theory B 44 (1988) 230-250.

[10] A.M. Frieze, On a Greedy 2-Matching Algorithm and Hamilton Cycles in Random Graphs with Minimum Degree at Least Three, submitted.

[11] A.M. Frieze, M.R.Jerrum, M.Molloy, R.Robinson and N.C.Wormald, Generating and counting Hamilton cycles in random regular graphs, Journal of Algorithms 21 (1996) 176-198.

[12] A.M. Frieze and B. Pittel, On a sparse random graph with minimum degree threee: Likely Posa's sets are large, submitted.

[13] Richard M. Karp, Reducibility among combinatorial problems, Complexity of computer computations (Proc. Sympos., IBM Thomas J. Watson Res. Center, Yorktown Heights, N.Y., 1972), Plenum, New York, 1972, pp. 85-103.

[14] R.M. Karp and M. Sipser, Maximum matchings in sparse random graphs, Proceedings of the 22nd Annual IEEE Symposium on Foundations of Computing (1981) 364-375.

[15] J. Komlós and E. Szemerédi, Limit distributions for the existence of Hamilton circuits in a random graph, Discrete Mathematics 43 (1983), 55-63.

[16] B. McKay, Asymptotics for 0-1 matrices with prescribed line sums, in Enumeration and Design, (Academic Press, 1984) 225-238.

[17] R.W. Robinson and N.C. Wormald, Almost all cubic graphs are Hamiltonian. Random Structures and Algorithms 3 (1992) 117-125.

[18] R.W. Robinson and N.C. Wormald, Almost all regular graphs are Hamiltonian. Random Structures and Algorithms 5 (1994) 363-374. 